\title{
Synergistic effects between $\kappa$-carrageenan and locust bean gum on physicochemical properties of edible films made thereof
}

\author{
Joana T. Martins a , Miguel A. Cerqueira ${ }^{a}$, Ana I. Bourbon ${ }^{a}$, Ana C. Pinheiro a , Bartolomeu W.S. Souza ${ }^{\text {a,b }}$, \\ António A. Vicente ${ }^{\mathrm{a}, *}$ \\ a IBB - Institute for Biotechnology and Bioengineering, Centre of Biological Engineering, Universidade do Minho, Campus de Gualtar, 4710-057 Braga, Portugal \\ ${ }^{\mathrm{b}}$ Departamento de Engenharia de Pesca, Universidade Federal do Ceará, Campus do Pici, Fortaleza, Ceará, Brazil
}

\section{A R T I C L E I N F O}

Article history:

Received 12 December 2011

Accepted 13 March 2012

\section{Keywords}

Food packaging

Biopolymers

Edible films

Synergistic interactions

Galactomannan

\begin{abstract}
A B S T R A C T
The development of mixed systems, formed by locust bean gum (LBG), and $\kappa$-carrageenan ( $\kappa$-car) can offer new interesting applications such as the development of edible films with particular properties. $\kappa$ car/LBG blend films with different ratios were developed, and their effects on films' physical properties were assessed. Thermogravimetric analysis (TGA), X-ray diffraction (XRD) patterns, dynamic mechanical analysis (DMA) and Fourier-transform infrared (FTIR) spectroscopy techniques were used to highlight the interactions between the two polysaccharides. The addition of $\kappa$-car to LBG improved the barrier properties of the films leading to a decrease of water vapor permeability (WVP). Improved values of elongation-at-break (EB) were registered when the ratio of $\kappa$-car/LBG was $80 / 20$ or $40 / 60(\% \mathrm{w} / \mathrm{w}$ ). Moreover, the $\kappa$-car/LBG blend films enhance the tensile strength (TS) compared to $\kappa$-car and LBG films. FTIR results suggested that hydrogen bonds interactions between $\kappa$-car and LBG have a great influence in films' properties e.g. moisture content, WVP. Therefore, different $\kappa$-car/LBG ratios can be used to tailor edible films with enhanced barrier and mechanical properties.
\end{abstract}

(c) 2012 Elsevier Ltd. All rights reserved.

\section{Introduction}

Biodegradable films and coatings prepared from various biological polymers such as polysaccharides, proteins or lipids have received great interest in recent years (Khwaldia, Arab-Tehrany, \& Desobry, 2010). Edible and biodegradable films can be used to inhibit/regulate the migration of moisture, oxygen, carbon dioxide, aromas, lipids, and also carry food ingredients to improve food properties (Bourtoom, 2008). Polysaccharide-based films have gained much interest and their physical properties have been extensively investigated (Mikkonen et al., 2007; Rao, Kanatt, Chawla, \& Sharmam, 2010). One approach to further improve films' properties has been the preparation of composite films produced from a combination of polysaccharides such as chitosan and galactomannans, combining the intrinsic properties of the different biopolymers (Rao et al., 2010). The addition of plasticizers (such as glycerol) to these polysaccharides can significantly

\footnotetext{
* Corresponding author. Tel.: +351 253604 419; fax: +351 253604429 .

E-mail addresses: joanamartins@deb.uminho.pt (J.T. Martins), miguelcerqueira@ deb.uminho.pt (M.A. Cerqueira), isabelbourbon@deb.uminho.pt (A.I. Bourbon), anapinheiro@deb.uminho.pt (A.C. Pinheiro), souzabw@gmail.com (B.W.S. Souza), avicente@deb.uminho.pt (A.A. Vicente).
}

improve their flexibility and are important to guarantee their processability (Mikkonen et al., 2007).

LBG, a non-ionic galactomannan naturally occurring in the seeds of some legumes, consists of a mannan backbone of $\beta-(1 \rightarrow 4)$-Dmannose with $\alpha$-D-galactose at C6 (Cerqueira et al., 2011). This biopolymer has the ability to form very viscous solutions at relatively low concentrations, and has been used to stabilize dispersions and emulsions and to replace fat in many dairy products (Pollard et al., 2007). This neutral polymer is only slightly soluble in cold water and requires heat to achieve full hydration and maximum viscosity (Dakia, Blecker, Robert, Wathelet, \& Paquot, 2008). It is also compatible with other gums and thickening agents such as carrageenan and xanthan to form a more elastic and stronger gel (Pinheiro et al., 2011).

Carrageenans are anionic linear sulfated polysaccharides composed of D-galactopyranose residues bonded by regularly alternating $\alpha-(1 \rightarrow 3)$ and $\beta-(1 \rightarrow 4)$ bonds. $\kappa$-car is one of the three main types of carrageenans, the others being I-carrageenan and $\lambda$-carrageenan. $\kappa$-car contains one negatively charged sulfate group and has 3,6-anhydro-D-galactopyranose residues in the chain that impart $\kappa$-car the ability to form gels (Thành et al., 2002).

$\kappa$-car and LBG mixtures are widely employed in numerous industrial food applications (García-García \& Totosaus, 2008). к-car 
and LBG, when mixed together, form a network whose strength depends on the preparation temperature and on the weight ratio between the two components (Pinheiro et al., 2011). $\kappa$-car and LBG by themselves are known for their gelling ability and good filmforming properties (Mikkonen et al., 2007; Sanchez-Garcia, Hilliou, \& Lagaron, 2010); however film-forming properties of $\kappa$-car/LBG mixtures have not been investigated. A new area of research on edible films could be created by studying synergies between polysaccharides (e.g. $\kappa$-car/LBG); it is expected that if such synergies occur they would possibly result from conformational changes and chemical interactions between the two polymers, which may result in improved physical properties.

The aims of the present work were to develop films composed by mixtures of $\kappa$-car and LBG and to assess the effect of different ratios of these polysaccharides on films' properties. This evaluation is carried out by analyzing the films' transport, mechanical and optical properties, being the chemical interactions between the two polysaccharides assessed by Fourier-transform infrared (FTIR) spectra, X-ray diffraction (XRD), dynamic mechanical analysis (DMA) and thermogravimetric analysis (TGA).

\section{Materials and methods}

\subsection{Material}

$\kappa$-car (Gelcarin DX5253) and LBG (Genu gum type RL-200) were supplied by FMC Biopolymer (Norway) and CP Kelco (USA), respectively. Other reagents for films formulations were glycerol 87\% (Panreac, Spain) and distilled water.

\subsection{Preparation of edible films}

Polysaccharide dispersions were prepared using the method described by Pinheiro et al. (2011). Briefly, polysaccharides ( $\kappa$-car and LBG) were suspended in distilled water under agitation during $1 \mathrm{~h}$ at $25^{\circ} \mathrm{C}$. The film-forming solutions were homogenized at $70^{\circ} \mathrm{C}$ under stirring during $30 \mathrm{~min}$ in the presence of $30 \%$ of glycerol $(\%$ $\mathrm{w} / \mathrm{w}$ of the total polysaccharide content) until a homogeneous solution was obtained. Glycerol concentration was constant in all film-forming solutions and was chosen based in preliminary work in our laboratory (data not shown) and following previous work (Karbowiak, Debeaufort, Champion, \& Voilley, 2006), where the lowest glycerol concentration needed for film formation was determined.

Polysaccharide mixtures were prepared by mixing appropriate amounts of $\kappa$-car and LBG to achieve a final polysaccharide concentration of $1 \%(\mathrm{w} / \mathrm{w})$ (Table 1$)$. Film-forming solutions were then degassed under vacuum to remove air bubbles and dissolved air as much as possible. Then, $28 \mathrm{ml}$ of the film-forming solution (at $70{ }^{\circ} \mathrm{C}$ to avoid the solution to turn into a gel at lower temperatures) was cast into polystyrene Petri dishes, and dried at $35^{\circ} \mathrm{C}$ during $16 \mathrm{~h}$. Films were conditioned at $54 \pm 1 \%$ relative humidity $(\mathrm{RH})$ and $20 \pm 1{ }^{\circ} \mathrm{C}$ by placing them in a desiccator containing a saturated solution of $\mathrm{Mg}\left(\mathrm{NO}_{3}\right)_{2} \cdot 6 \mathrm{H}_{2} \mathrm{O}$.

Table 1

Experimental design of composite films with $\kappa$-car/LBG different mixtures.

\begin{tabular}{|c|c|c|c|}
\hline$\kappa$-car/LBG film (\% w/w) & $\kappa-\operatorname{car}(\% \mathrm{w} / \mathrm{w})$ & LBG $(\% \mathrm{w} / \mathrm{w})$ & Glycerol (\% w/w) \\
\hline $100 / 0$ & 100 & 0 & 30 \\
\hline $80 / 20$ & 80 & 20 & 30 \\
\hline $60 / 40$ & 60 & 40 & 30 \\
\hline $40 / 60$ & 40 & 60 & 30 \\
\hline $20 / 80$ & 20 & 80 & 30 \\
\hline $0 / 100$ & 0 & 100 & 30 \\
\hline
\end{tabular}

\subsection{Film characterization}

\subsubsection{Fourier-transform infrared (FTIR) spectroscopy}

The FTIR spectra of the films were recorded with an infrared spectrometer (Perkin-Elmer 16 PC spectrometer, Boston, USA), using Attenuated Total Reflectance mode (ATR). Each spectrum results from 16 scans in the wavelength range $400-4000 \mathrm{~cm}^{-1}$. Signal averages were obtained at a resolution of $4 \mathrm{~cm}^{-1}$ (Pereira, Souza, Cerqueira, Teixeira, \& Vicente, 2010).

\subsubsection{X-ray diffraction (XRD)}

XRD patterns of $\kappa$-car/LBG films were obtained using a Bruker D8 Discover (USA)X-ray diffractometer $(40 \mathrm{kV}, 40 \mathrm{~mA})$ equipped with $\mathrm{Cu}$ radiation at wavelength of $0.154 \mathrm{~nm}(1.5406 \AA)$. All experiments were carried out with a scanning rate of $0.04^{\circ} / \mathrm{min}$ from 6 to $40^{\circ}$ ( $2 \theta$ range).

\subsubsection{Dynamic mechanical analyses (DMA)}

The dynamic mechanical measurements were conducted using a Tritec 2000 DMA (Triton Technology, UK) operating in the tensile mode. The film samples for DMA analysis were cut into $5 \mathrm{~mm} \times 30 \mathrm{~mm}$ strips and fixed in a grip probe. The width of the samples was $5.5 \mathrm{~mm}$ and their average thickness was determined as described in Section 2.3.5. The samples were heated at $3^{\circ} \mathrm{C} \mathrm{min}^{-1}$ between -70 and $200^{\circ} \mathrm{C}$. A dynamic deformation was applied at a frequency of $1 \mathrm{~Hz}$ under a strain amplitude of $4 \mu \mathrm{m}$. The viscoelastic properties obtained were the storage modulus $\left(E^{\prime}\right)$, loss modulus $\left(E^{\prime \prime}\right)$ and $\tan \delta\left(=E^{\prime \prime} \mid E^{\prime}\right)$. The measurements were conducted at least in duplicate with the $T_{\mathrm{g}}$ values measured as the peak temperature of $\tan \delta$. All film samples were conditioned at $54 \pm 1 \% \mathrm{RH}$ and $20 \pm 1{ }^{\circ} \mathrm{C}$ before analysis.

\subsubsection{Thermogravimetric analyses (TGA)}

Thermal stability and degradation of films were analyzed by thermogravimetric analyses (TGA), performed with a Shimadzu TGA-50 (Shimadzu Corporation, Kyoto, Japan). Samples were placed in the balance system and heated from $20{ }^{\circ} \mathrm{C}$ to $580{ }^{\circ} \mathrm{C}$ at a heating rate of $10^{\circ} \mathrm{C} \mathrm{min}^{-1}$ under a nitrogen atmosphere to avoid thermo-oxidative reactions (Casariego et al., 2009).

\subsubsection{Thickness}

A digital micrometer (No. 293-5, Mitutoyo, Japan) was used to measure the film thickness. Five thickness measurements were randomly taken on each film sample and the average was used to calculate water vapor permeability and tensile strength.

\subsubsection{Moisture content}

The moisture content (MC) was expressed as the percentage of water removed from the initial mass sample. MC was determined gravimetrically by drying film samples (of about $20 \mathrm{mg}$ ) at $105^{\circ} \mathrm{C}$ in an oven with forced air circulation for $24 \mathrm{~h}$. The experiments were performed on each film sample in triplicate.

\subsubsection{Water vapor permeability (WVP)}

WVP was determined gravimetrically using the ASTM E96-92 method described by Casariego et al. (2009). Three samples were cut from each film. Each sample was sealed on a permeation cell (cup containing distilled water at $100 \% \mathrm{RH} ; 2.337 \times 10^{3}$ Pa vapor pressure at $20^{\circ} \mathrm{C}$ ), and placed in a desiccator containing silica gel $\left(0 \% \mathrm{RH} ; 20^{\circ} \mathrm{C}\right)$. The water transferred through the test films was determined from cup weight loss over time. The cups were weighed to the nearest $0.1 \mathrm{mg}$ at $2 \mathrm{~h}$ intervals. The steady state of weight loss was reached after $10 \mathrm{~h}$. The experiments were performed in triplicate for each film formulation.

\subsubsection{Tensile strength (TS) and elongation-at-break (EB)}

Tensile strength (TS) and elongation-at-break (EB) were determined with an Instron Universal Testing Machine (Model 4500, 
Instron Corporation) using ASTM Standard Method D 882-91 (Pereira et al., 2010). Film specimens $\left(45 \times 20 \mathrm{~mm}^{2}\right.$ strips $)$ were cut from each preconditioned ( $54 \% \mathrm{RH}$ ) film and placed between the tensile grips. The initial grip separation and crosshead speed were set to $30 \mathrm{~mm}$ and $5 \mathrm{~mm} / \mathrm{min}$, respectively. TS was expressed in MPa and EB was expressed in percentage. Five samples for each type of films were replicated.

\subsubsection{Optical properties}

Optical measurements were performed in a Minolta colorimeter (Minolta CR 400, Tokyo, Japan). The CIELab scale was used to determined $L^{*}, a^{*}$ and $b^{*}$ parameters (Martins, Cerqueira, Souza, Avides, \& Vicente, 2010) Standard values considered were those of a white background ( $L^{*}=84.67, a^{*}=-0.55$, and $\left.b^{*}=0.68\right)$. The opacity of the samples was determined according to the Hunterlab method, as the relationship between the opacity of each sample on a black standard and the opacity of each sample on a white standard. Six measurements were taken of each sample, and three samples of each film were tested.

\subsubsection{Optical microscopy}

Microscopic observations of the film samples were carried out with an optical microscope (Leitz Laborlux S, Germany) to which a digital camera (Olympus Altra-20) was attached for image recording. The film samples were evaluated at $100 \times$ magnification. The images were processed with image analysis software (Analysis getIT, Olympus).

\subsection{Statistical analyses}

Statistical analyses were performed with Microsoft Windows Excel 2003 and software SigmaPlot 11.0 for Windows. One-way analysis of variance and Tukey's multiple comparisons test were performed to determine the significance of differences, defined for $p<0.05$. The relationship between moisture content, WVP, mechanical and optical properties was studied using explorative principal component analysis (PCA) with R software for Windows (version 2.11.1).

\section{Results and discussion}

\subsection{Fourier-transform infrared (FTIR) spectroscopy}

Infrared spectroscopy is a rapid and a non-destructive technique that has been widely used to characterize different polysaccharides (Turquois, Acquistapace, Vera, \& Welti, 1996). Also, FTIR spectroscopy is a powerful technique to investigate polymer blend miscibility. When chemical groups interact at the molecular level, changes are seen in FTIR spectra such as the shifting of absorption bands. These changes can be an indication of good miscibility of polymers (Xu, Li, Kennedy, Xie, \& Huang, 2007). FTIR analyses were used to evaluate the interaction between $\kappa$-car and LBG in $\kappa$-car/ LBG films (Fig. 1a). The broad band ranging between 3500 and $3100 \mathrm{~cm}^{-1}$ was attributed to $\mathrm{O}-\mathrm{H}$ stretching vibration formed by the hydroxyl group of polysaccharides and water and the broad band around $2800-3000 \mathrm{~cm}^{-1}$ was attributed to $\mathrm{C}-\mathrm{H}$ stretching vibration (Cerqueira et al., 2011).

FTIR spectra of our samples also show a band in the region of $750-1300 \mathrm{~cm}^{-1}$ that corresponds to the carbohydrates region (Fig. 1b). These wavenumbers are within the so-called fingerprint region, where the bands are specific for each polysaccharide, allowing its possible detection (Sen \& Erboz, 2010). In particular, the spectrum of 100/0 $\mathrm{\kappa}$-car/LBG films shows several peaks (Fig. 1b), from which it is important to highlight a peak at $1220 \mathrm{~cm}^{-1}$ corresponding to the ester sulfate groups, a peak at $922 \mathrm{~cm}^{-1}$ attributed to the 3,6-anhydrogalactose group, a peak at $846 \mathrm{~cm}^{-1}$ corresponding to galactose-4-sulfate and a peak at $805 \mathrm{~cm}^{-1}$ corresponding to 3,6-anydro-D-galactose-2-sulfate that indicates the presence of impurities in the carrageenan samples (Pereira, Amado, Critchley, van de Velde, \& Ribeiro-Claro, 2009; Turquois et al., 1996). In turn, the spectrum of $0 / 100 \mathrm{\kappa}$-car/LBG films shows absorption bands at $817 \mathrm{~cm}^{-1}$ and $873 \mathrm{~cm}^{-1}$ indicating the presence of $\alpha$ linked D-galactopyranose units and $\beta$-linked D-mannopyranose units, respectively (Cerqueira et al., 2011; Figueiró, Góes, Moreira, \& Sombra, 2004). Fig. 1b also showed the decrease of the intensity of bands at $817 \mathrm{~cm}^{-1}$ and $873 \mathrm{~cm}^{-1}$, characteristic of LBG, when the $\kappa$ car is added to the film composition. In the same way, $\kappa$-car characteristic intensity bands at $846 \mathrm{~cm}^{-1}$ and at $1204-1295 \mathrm{~cm}^{-1}$ decrease as LBG ratio increase.

The chemical interaction between the two polysaccharides can be observed by the shift in the peak position of their main groups (Wanchoo \& Sharma, 2003). In the spectra of these $\kappa$-car/LBG films it is possible to observe shifts in characteristic peaks, such as those corresponding to $\mathrm{C}-\mathrm{O}$ stretching band of the $\mathrm{C}-\mathrm{O}-\mathrm{H}$ group band

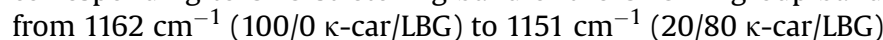
that can be related to the interaction (possibly through hydrogen bridges) of the OH groups of LBG with $\kappa$-car structure (Ning, Jiugao, Xiaofei, \& Ying, 2007). The shifts of two other peaks were also observed: from $1023 \mathrm{~cm}^{-1}$ (0/100 $\mathrm{\kappa}$-car/LBG) to $1033 \mathrm{~cm}^{-1}(80 / 20$ $\kappa$-car/LBG) and from $919 \mathrm{~cm}^{-1}\left(100 / 0 \kappa\right.$-car/LBG) to $926 \mathrm{~cm}^{-1}(20 /$ $80 \kappa-\mathrm{car} / \mathrm{LBG})$, which correspond to the $\mathrm{C}-\mathrm{O}$ stretching of the C-O-C group, respectively (Ning et al., 2007; Pelissari, Grossmann, Yamashita, \& Pineda, 2009). This last shift has been related with the involvement of the $\mathrm{C}-\mathrm{O}$ in the interactions between the two polysaccharides (Ning et al., 2007).

This type of behavior (e.g. changing of the stretching frequencies of groups participating in interactions - such as $\mathrm{O}-\mathrm{H}$ and $\mathrm{C}-\mathrm{O}$ ) is a pattern of miscible blends being the change (shift) of peak position dependent of the strength of the interaction (Wanchoo \& Sharma, 2003). Possibly, $\kappa$-car could interact through hydrogen bonding with unbranched smooth segments of the D-mannose backbone of LBG molecule (Tako, Qi, Yoza, \& Toyama, 1998).

The present results suggest physical entanglements and miscibility between these two polysaccharides (Figueiró et al., 2004; Sen \& Erboz, 2010) showing that the interaction between $\kappa$-car and LBG occurred and possibly may lead to changes in films' transport, thermal and mechanical properties.

\section{2. $X$-ray diffraction (XRD)}

Polysaccharides naturally interact with water leading to waterinduced structural transitions (related to amorphous-crystalline transitions), having a great impact on their molecular mobility and functional properties (Yakimets et al., 2007).

Fig. 2 shows the X-ray diffraction spectra of the analyzed films. The film composed of LBG (0/100 $\kappa$-car/LBG) shows a typical noncrystalline structure, presenting a broad peak around $2 \theta=20^{\circ}$ (Fig. 2a), characteristic of amorphous structures. Similar results were observed for konjac glucomannan-based films, for cassava starch-based films and for a guar gum solution (Cunha, Castro, Rocha, de Paula, \& Feitosa, 2005; Li et al., 2011; Yakimets et al., 2007). Fig. 2b shows the X-ray spectrum for the $\kappa$-car film (100/ $0 \kappa$-car/LBG) indicating that the molecules of this polysaccharide do not organize into microcrystallites. However, it is evident the presence of one sharp peak $\left(2 \theta=28.4^{\circ}\right)$ probably due to inorganic salts such as $\mathrm{KCl}$ present in the $\kappa$-car film sample (Prasad, Kaneko, \& Kadokawa, 2009) strongly suggesting that the crystallinity was not due to $\kappa$-car itself. A change in XRD pattern was observed when LBG was added; the sharp peak at $28.4^{\circ}$ disappeared and only the broad amorphous band was observed. The peak disappears when LBG is 

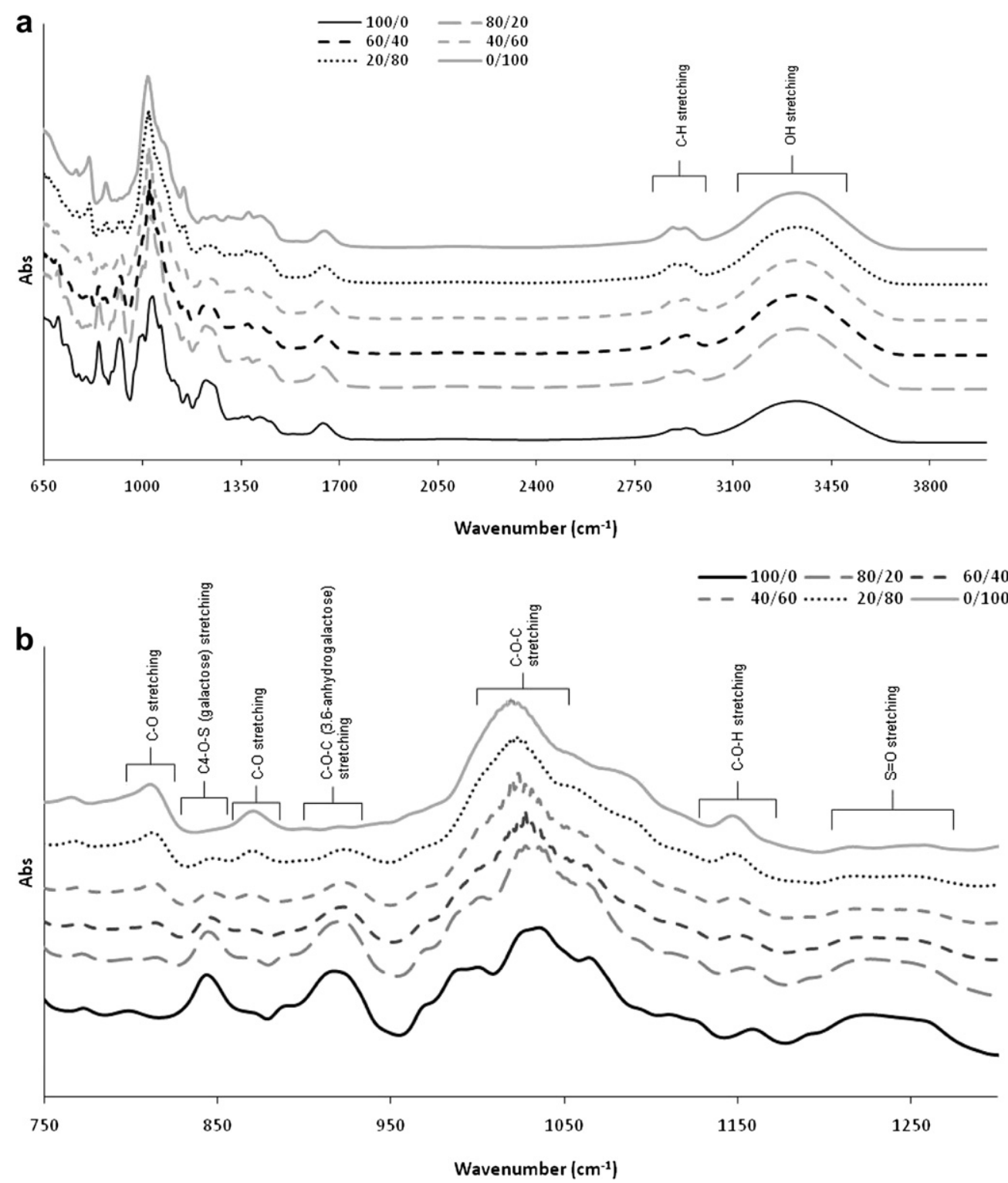

Fig. 1. Fourier-transform infrared (FTIR) spectra of the $\kappa$-car/LBG films between 650 and $4000 \mathrm{~cm}^{-1}$ (a) and $750-1300 \mathrm{~cm}^{-1}$ (b).

added to the $\kappa$-car once the salts could be involved in the interaction developed between both polysaccharides. Potassium ions have specific site-binding to $\kappa$-car, which has an impact on the aggregation and gel formation of $\kappa$-car molecules (Williams, 2009). Being so it would also influence $\kappa$-car-LBG interaction. When LBG is added to the $\kappa$-car intermolecular interaction may occur between the ring oxygen atom of the former and $\mathrm{OH}-2$ of the D-mannopyranosyl residue with hydrogen bonding, at which the intramolecular $\mathrm{K}^{+}$bridge is inserted into the adjacent unsubstituted segments of the backbone of the LBG, as proposed by Tako et al. (1998). At the molecular level, it is known that the formation of a $\kappa$-car gel is preceded by a disorder-order transition of polysaccharide conformation, followed by aggregation of helices which gives rise to low crystalline materials, not detected by XRD (Johnson, Busk, \& Labuza, 1980).

All the blend compositions show a broad and diffuse peak, which indicates the amorphous nature of the blends. These results are in agreement with previous studies, where new ordered (crystalline) structure in $\kappa$-car/LBG mixture were not observed when using XRD (Parker, Lelimousin, Miniou, \& Boulenguer, 1995). On the other hand, Williams and Langdon (1996) observed the interaction between $\kappa$-car/LBG using differential scanning calorimetry (DSC), which implied a crystalline structure, where the LBG chains take part in a direct association process involving bundles of self-aggregated $\kappa$-car. These authors pointed out that this mechanism does not compromise the XRD data, however the conformational state of the macromolecules still is a matter of debate (Parker et al., 1995).

A slight shoulder around $2 \theta=10.6^{\circ}$ was enhanced in $20 / 80 \kappa-$ car/LBG films when comparing with 0/100 $\kappa$-car/LBG films, indicating a slight phase separation between LBG and $\kappa$-car. Zhong and Xia (2008) also observed that peak intensity around $2 \theta=10^{\circ}$ was enhanced when cassava starch was added to chitosan films. Additionally, the diffraction peak of the blends films at $2 \theta=20^{\circ}$ became broader when compared with that obtained for $\kappa$-car and LBG, which means that there is good compatibility between both polymers (Abugoch, Tapia, Villamán, Yazdani-Pedram, \& Díaz-Dosque, 2011). 
Also, optical microscopy images show differences in films' structure (Fig. 1 in Supplementary data). All the films were optically clear and appear to be homogeneous, however some differences can be observed: 100/0 $\mathrm{\kappa}$-car/LBG films showed the presence of crystalline aggregates, which is in agreement with XRD patterns; 40/60 and 0/100 $\kappa$-car/LBG films exhibited an amorphous appearance without an organized structure.

\subsection{Dynamic mechanical analysis (DMA)}

DMA was used to determine the glass transition temperature $\left(T_{\mathrm{g}}\right)$ of film samples. $T_{\mathrm{g}}$ is the region where the transition from glassy to rubbery deformation occurs. $T_{\mathrm{g}}$ is often used as a criterion to assess the miscibility of the polymer blend: a single transition between $T_{\mathrm{g}}$ of the two polymers indicates a good miscibility, whereas separate transitions of the mixture indicates immiscibility of their components (Biliaderis, Lazaridou, \& Arvanitoyannis, 1999).

Fig. 3 shows $E^{\prime}$ and $\tan \delta$ spectra from the DMA for the films 100/ $0 \kappa$-car/LBG, 40/60 $\kappa$-car/LBG and 0/100 $\kappa$-car/LBG. In this study, $T_{\mathrm{g}}$ was defined as a peak in $\tan \delta$ within the decreasing range of $E^{\prime}$. Only the film composed of $0 / 100 \kappa$-car/LBG presents a distinct $T_{\mathrm{g}}$, with a value of $5.2{ }^{\circ} \mathrm{C}$, which is related to the collective motion of molecules in the polymer phase (Mikkonen et al., 2007). It is known that molecular motion of the materials observed at the $T_{\mathrm{g}}$ is not the
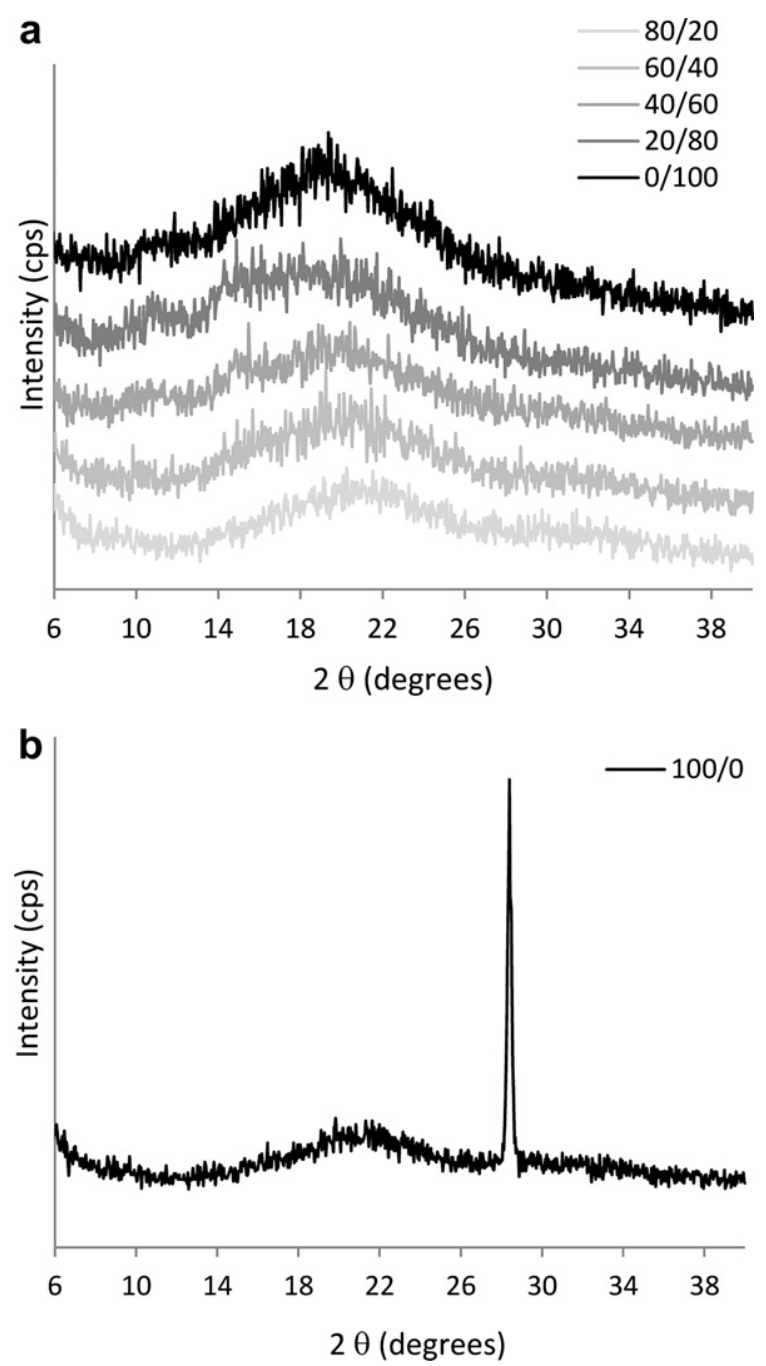

Fig. 2. X-ray diffraction pattern of $\kappa$-car/LBG mixtures (a) and for the $\kappa$-car (100/0) films (b). only deformation mechanism that can occur in amorphous and semi-crystalline materials (such as polymeric carbohydrates, proteins and other polymers) (Duncan, 2008). The first secondary relaxation below $\alpha$-relaxation $\left(T_{\mathrm{g}}\right)$ is referred to as $\beta$-relaxation and is related with the local motions within polymeric chains (Jones, 1999). In our work, a $\beta$-relaxation can be observed at $-47.6{ }^{\circ} \mathrm{C}$. This is probably due to the relaxation associated with the glass transition of glycerol $\left(-75{ }^{\circ} \mathrm{C}\right)$, as mentioned by other authors (Avérous, Fringant, \& Moro, 2001; Mathew \& Dufresne, 2002). The obtained values are in agreement with the values reported by other authors for guar gum and LBG-based films (Mikkonen et al., 2007). It is worth noting the atypical pattern of $E^{\prime}$ in the range -20 to $80^{\circ} \mathrm{C}$, clearly evident for the $0 / 100$ film sample; where the $E^{\prime}$ curve first decreases reaching to a minimum, and then suffers an increase. The decrease can be explained by the water crystallisation/formation of ice crystals that at temperatures below $0{ }^{\circ} \mathrm{C}$ will reinforce
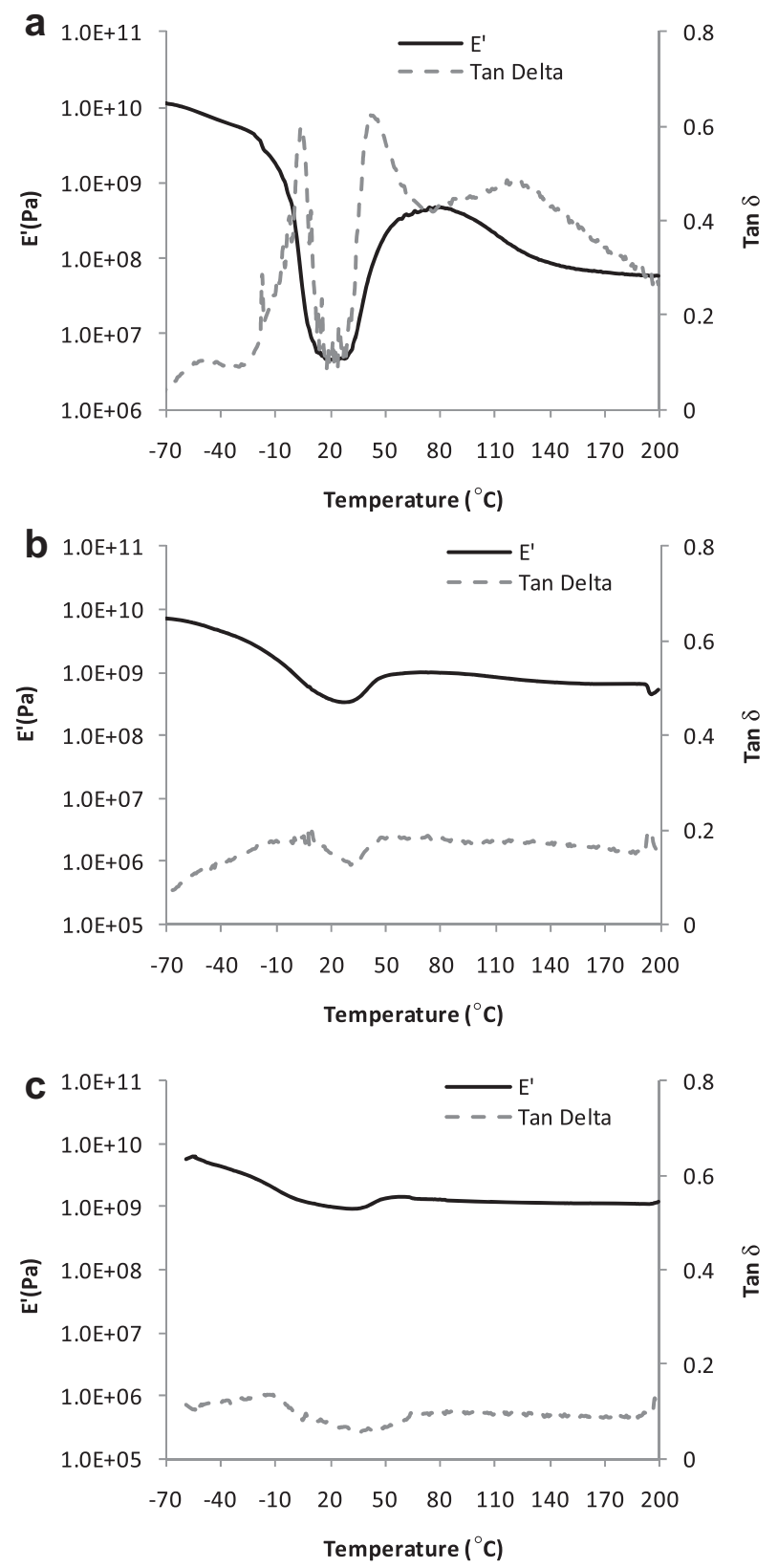

Fig. 3. DMA plot for LBG (0/100) (a), 40/60 $\kappa$-car/LBG (b) and $\kappa$-car (100/0) (c) films. 
the biopolymer structure. As temperature increases, crystals begin to melt and consequently material stiffness decrease (Mano, 2005); that explains the decrease of $E^{\prime}$ below $0{ }^{\circ} \mathrm{C}$. The broad $\tan \delta$ peak (at $5{ }^{\circ} \mathrm{C}$ ) between -30 and $20^{\circ} \mathrm{C}$, may be attributed to melting of water and glass transition (increased molecular mobility of polysaccharide chains) (Vittadini \& Vodovotz, 2003). At $40{ }^{\circ} \mathrm{C}$, an intense peak was observed for $\tan \delta$, along with a corresponding storage modulus increase. This fact could be due to the stiffening effect provoked by the loss of water that is trapped in the biopolymer (Cherian, Gennadios, Weller, \& Chinachoti, 1995). Films with $\kappa$-car as component do not present a distinct $T_{\mathrm{g}}$; however a relaxation step can be observed at temperatures of 9.8 and $-11.8^{\circ} \mathrm{C}$ for $40 / 60 \kappa$-car/LBG and $0 / 100 \kappa$-car/LBG, respectively. The position of $\alpha$ relaxation of LBG in $\tan \delta$ curve has changed only slightly, but the peak became less prominent with increasing $\kappa$-car concentration (Fig. 3b and c). Liu, Dever, Fair, and Benson (1997) reported that amorphous polymers usually present more intense and sharp $\tan \delta$ peaks, once motion restriction decreases with a decrease in the crystallinity of the polymer. The reduced magnitude of $\tan \delta$ peak for $100 / 0 \kappa$-car/LBG films can be attributed to crystalline regions that inhibit the chain mobility in the amorphous regions. From these results it is not possible to assess if $\kappa$-car and LBG form miscible blends because no distinct $T_{\mathrm{g}}$ was detected in films containing $\kappa$-car. Thus, DMA proved inadequate to evaluate the miscibility of the $\kappa$-car and LBG blend and other techniques such as FTIR had to be used.

\subsection{Thermogravimetric analysis (TGA)}

TGA was performed to evaluate the stability of the films and to determine how the interaction between the two polysaccharides could influence stability. TGA curves (Fig. 4) show the thermal events for all $\kappa$-car/LBG films. The derivative of weight loss (DTG) curves of all films showed at least three significant thermal events (Fig. 5). Thermal analyses show that films are stable up to $60^{\circ} \mathrm{C}$ for all the formulations (Fig. 4). The first stage $\left(60-120{ }^{\circ} \mathrm{C}\right)$ may be attributed to water evaporation, and chemisorbed water through hydrogen bonds. The second stage $\left(170-230{ }^{\circ} \mathrm{C}\right)$, is usually attributed to the presence of glycerol (Cerqueira, Souza, Teixeira, \& Vicente, 2012). The third stage at $230-330{ }^{\circ} \mathrm{C}$ (maximum peak in the DTG curve) is related to polysaccharide decomposition (Zohuriaan \& Shokrolahi, 2004). The 0/100 films suffered a weight

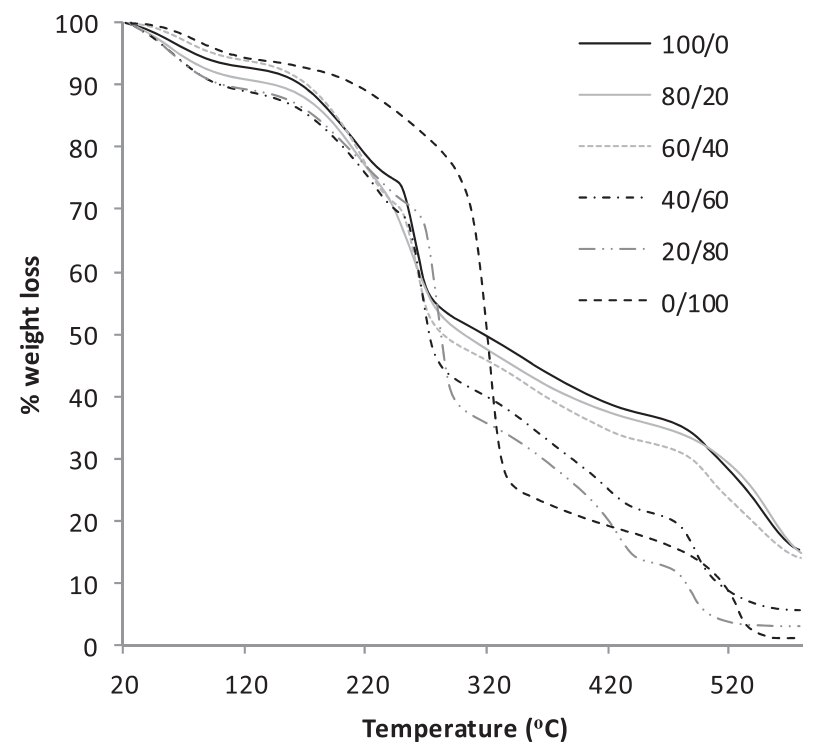

Fig. 4. TGA thermograms of $\kappa$-car/LBG films at different ratios.

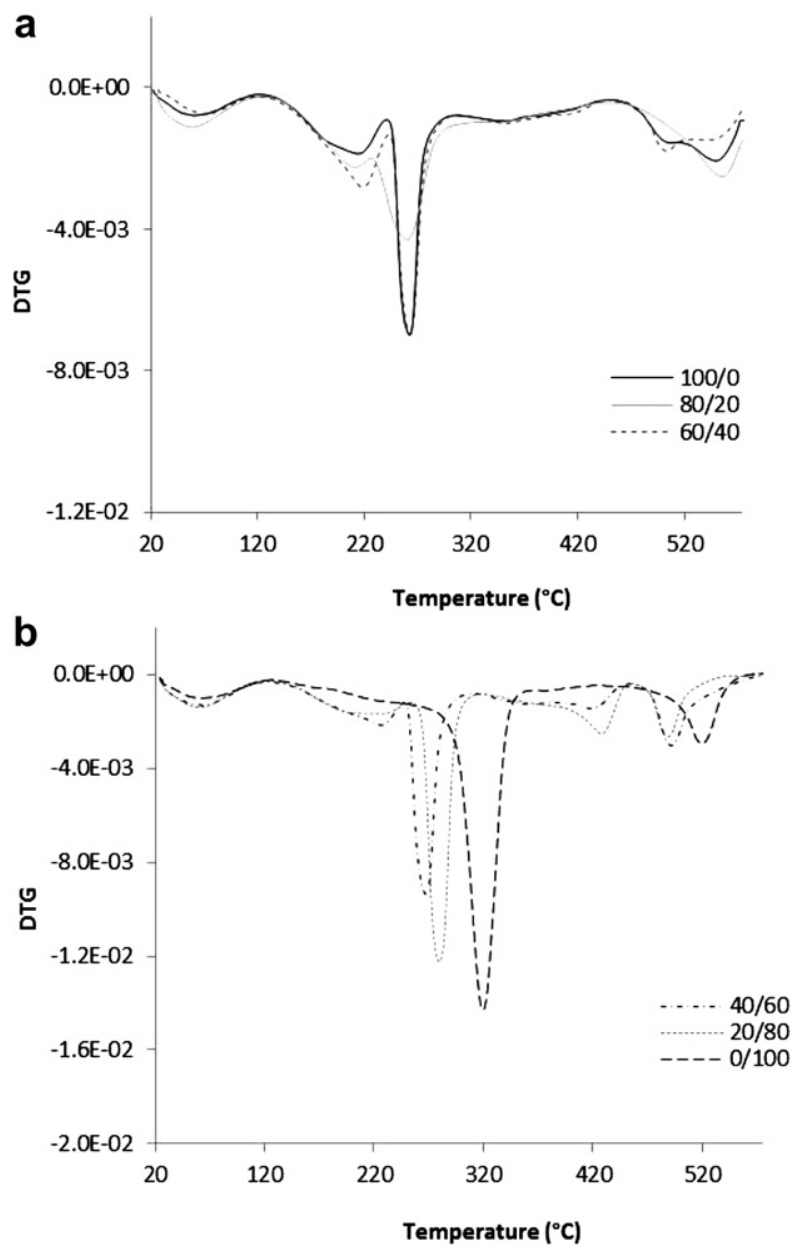

Fig. 5. Derivative thermogravimetric (DTG) curves of (a) 100/0, 80/20, 60/40; (b) 40/ $60,20 / 80$ and $0 / 100 \kappa-c a r / L B G$ films.

loss of ca. 55\% at a DTG of $319.2{ }^{\circ} \mathrm{C}$, due to LBG thermal decomposition. These values are in conformity with the values reported by Vendruscolo et al. (2009) for the galactomannan of Mimosa scabrella, with values ranging between 299.7 and $311.9{ }^{\circ} \mathrm{C}$. Additionally, the third thermal event of 0/100 $\mathrm{\kappa}$-car/LBG films happens at a higher temperature when compared with the other samples (Fig. 5), indicating that $0 / 100 \kappa$-car/LBG films present a higher thermal stability when compared with other films where $\kappa$-car is present. This is possibly caused by the presence of charged groups in $\kappa$-car molecules as compared to the neutral molecules of LBG. According to Villetti et al. (2002) in general, neutral polysaccharides showed higher thermal resistance than charged polysaccharides. A similar behavior has been reported for other charged biopolymers, when compared to xanthan and starch (Soares, Lima, Oliveira, Pires, \& Soldi, 2005). After degradation at stage three, all samples showed a progressive decrement in weight up to $540{ }^{\circ} \mathrm{C}$ attributed to the further breakage of products of the composition during the third stage (Fig. 4). Once FTIR results showed that different film blends presented different interactions $(\mathrm{C}-\mathrm{O}, \mathrm{OH})$, this could mean that the different $\kappa$-car/LBG ratios might lead to diverse decomposition mechanisms and, consequently, display different thermal stabilities.

\subsection{Moisture content}

Film moisture content provides information about how the interaction between $\kappa$-car and LBG could affect the water affinity of 
edible films. Table 2 shows the water content of $\kappa$-car/LBG films with different ratios. Results show that the addition of LBG (and the corresponding decrease of $\kappa$-car) lead to an increase of water content $(p<0.05$ ) from $19.28 \%$ to $26.77 \%$ (100/0 and $80 / 20 \kappa$-car/ LBG, respectively). Possibly, in $100 / 0 \kappa$-car/LBG films there is a formation of cross-linking between the chains of $\kappa$-car polymer (Watanabe, Ohtsuka, Murase, Barth, \& Gersonde, 1996); crosslinking induces a decrease in the availability of hydroxyl groups, limiting polysaccharide-water interactions by hydrogen bonding. It had been reported that the addition of galactomannans increases the water-binding capability of $\kappa$-car but then decreases by a further increase in the galactomannan concentration (Arda, Kara, \& Pekcan, 2009). The interaction between $\kappa$-car and LBG at 80/20 $\kappa-$ car/LBG ratio could lead to a higher susceptibility of the films to water, probably due to the exposure of hydroxyl groups. However, when the percentage of LBG tended to be higher than $\kappa$-car (from $40 / 60$ to $20 / 80 \kappa$-car/LBG), water content in the film decreased $(p<0.05)$. The lowest percentage of moisture content was found for 0/100 $\kappa$-car/LBG. Possibly, $\kappa$-car addition imparts a higher hydrophilicity to the films caused by the presence of sulfate groups (Xu, Bartley, \& Johnson, 2003). From these results, it is apparent that varying the concentration of $\kappa$-car can result in modification of the network structure of the films, which is reflected in the moisture content. These values of moisture content are in the range of those obtained for other polysaccharide-based films (Garcia, Pinotti, \& Zaritzky, 2006; Ziani, Oses, Coma, \& Maté, 2008).

\subsection{Water vapor permeability (WVP)}

The WVP of edible films depends on many factors, such as the integrity of the film, the hydrophilic-hydrophobic ratio, the ratio between crystalline and amorphous zones, and the polymeric chain mobility (Souza, Cerqueira, Teixeira, \& Vicente, 2010). One of the major purposes of edible films is to block moisture transfer between the food and the surrounding atmosphere, therefore the WVP should be as low as possible. Permeability is the contribution of diffusivity and solubility of the permeant through the solid matrix, being this parameters changed by the structure of the matrix when using the same permeant. Table 2 reports the WVP values of $\kappa$-car and LBG films at different ratios. Films containing only $\kappa$-car $(100 / 0 \kappa$-car/LBG) exhibit lower $(p<0.05)$ WVP than those containing only LBG $(0 / 100 \kappa$-car/LBG). These differences between $\kappa$-car and LBG-based films may find an explanation in the differences of amorphous-crystalline structure of the films. It is known that the permeability is primarily a function of the amorphous phase, being the crystalline phase assumed as impermeable (Souza et al., 2010). The presence of salts in the film matrix, as demonstrated by XRD, could lead the $100 / 0 \kappa$-car/LBG films to present lower WVP values.

The formation of films with a ratio $80 / 20 \kappa$-car/LBG leads to an increase of WVP values, but when the LBG ratio increases a subsequent decrease or stabilization of the values were observed. The differences in WVP for different $\kappa$-car/LBG ratios may be attributed to differences in film gel formation and interactions between $\kappa$-car and LBG. At elevated temperatures $\left(>50{ }^{\circ} \mathrm{C}\right.$, depending on salt concentration) $\kappa$-car exists in solution as a random coil conformation (Spagnuolo, Dalgleish, Goff, \& Morris, 2005; van de Velde \& de Ruiter, 2005), but a transition to a helix conformation occurs after cooling leading in most cases to aggregation and gelation (Karbowiak, Debeaufort, et al., 2006). Once film drying was conducted at $35{ }^{\circ} \mathrm{C}$, $\kappa$-car molecules could change from coil to helix conformation. The films formed could present a more compact matrix, which may be attributed to $\kappa$-car helices' association in the film structure (Karbowiak, Hervet, et al., 2006). Previous studies proved that $\kappa$-car helices association was formed in solid state (Karbowiak, Hervet, et al., 2006; Rees, 1981). Additionally, as the samples became colder and eventually reached their gelation temperatures, $\kappa$-car began to form double helices and junction zones which produce by hydrogen bonding of adjacent double helixes (Rees, 1981). Consequently, the $\kappa$-car chains were able to form a three dimensional network before complete evaporation of water. The chains then became locked into this structure as the solution dried into a film. This is a hypothesis that needs to be confirmed by further work. This can also suggest a network formation between $\kappa$-car and LBG structures that leads to a more compact film matrix thus improving the barrier to water vapor. FTIR analysis showed that changes in the film composition could be the reason for the differences found in the WVP values and the detected shifts of peak bands suggest the strengthening of chemical bonds. Similar results were observed by other authors for mixtures of polysaccharides such as agar, cassava starch and arabinoxylan (Phan The, Debeaufort, Voilley, \& Luu, 2009). The obtained values are in agreement with reported results for carrageenan and galactomannan-based films (Martins et al., 2010; Sanchez-Garcia et al., 2010).

\subsection{Mechanical properties - TS and EB}

Mechanical strength and extensibility are generally required for a film to resist external stress and maintain its integrity when applied to food products. The effect of the ratio of $\kappa$-car/LBG on the mechanical properties of the films is shown in Table 2. LBG films present significantly $(p<0.05)$ higher EB and lower TS values when compared with $\kappa$-car films. 100/0 $\kappa$-car/LBG films exhibited TS values comparable to $\kappa$-car films produced by other authors (Cha, Choi, Chinnan, \& Park, 2002). The differences between EB and TS values of $0 / 100 \kappa$-car/LBG and $100 / 0 \kappa$-car/LBG films can be explained by the different relative amounts of amorphous and crystalline parts of the samples (Ziani et al., 2008). 100/0 K-car/LBG films presented higher TS and lower EB values, probably due to the presence of crystalline structure in the film matrix as demonstrated by XRD. The film showing the lowest values of EB and TS $(p<0.05)$ was the one composed of 20/80 $\kappa$-car/LBG. On the other hand, $40 /$ $60 \kappa$-car/LBG films have exhibited the highest value of TS (27.57 MPa). The benefit of this combination is the improvement in the TS for $\kappa$-car and LBG films. An increase of TS has been also

Table 2

Values of thickness, moisture content (MC), water vapor permeability (WVP), elongation-at-break (EB) and tensile strength (TS) obtained for $\kappa$-car/LBG films.

\begin{tabular}{|c|c|c|c|c|c|}
\hline$\kappa-\operatorname{car} /$ LBG $(\% \mathrm{w} / \mathrm{w})$ & Thickness (mm) & Moisture content (\%) & $\mathrm{WVP} \times 10^{-11}\left(\mathrm{~g}\left(\mathrm{~m} \mathrm{~s} \mathrm{~Pa}^{-1}\right)\right.$ & EB (\%) & $\mathrm{TS}(\mathrm{MPa})$ \\
\hline $100 / 0$ & $0.052 \pm 0.001^{\mathrm{a}}$ & $19.28 \pm 0.58^{\mathrm{a}}$ & $5.48 \pm 0.19^{\mathrm{abc}}$ & $16.18 \pm 1.57^{\mathrm{a}}$ & $19.95 \pm 1.06^{\mathrm{a}}$ \\
\hline $80 / 20$ & $0.039 \pm 0.004^{b}$ & $26.77 \pm 1.37^{b}$ & $6.32 \pm 0.26^{\mathrm{d}}$ & $22.06 \pm 2.57^{b}$ & $20.68 \pm 1.97^{\mathrm{ab}}$ \\
\hline $60 / 40$ & $0.043 \pm 0.003^{b}$ & $24.81 \pm 0.42^{\mathrm{b}}$ & $6.00 \pm 0.30^{\mathrm{ad}}$ & $14.95 \pm 1.18^{\mathrm{a}}$ & $22.98 \pm 1.91^{\mathrm{b}}$ \\
\hline $40 / 60$ & $0.042 \pm 0.002^{\mathrm{b}}$ & $21.08 \pm 1.13^{\mathrm{ab}}$ & $5.15 \pm 0.11^{b}$ & $19.88 \pm 1.44^{\mathrm{b}}$ & $27.57 \pm 1.46^{\mathrm{c}}$ \\
\hline $20 / 80$ & $0.049 \pm 0.003^{a}$ & $19.25 \pm 0.21^{\mathrm{a}}$ & $5.43 \pm 0.06^{\mathrm{c}}$ & $10.19 \pm 3.40^{c}$ & $18.19 \pm 0.83^{\mathrm{d}}$ \\
\hline $0 / 100$ & $0.061 \pm 0.003^{c}$ & $13.69 \pm 0.63^{c}$ & $8.01 \pm 0.40^{\mathrm{e}}$ & $28.21 \pm 2.89^{d}$ & $8.61 \pm 3.27^{e}$ \\
\hline
\end{tabular}

${ }^{\mathrm{a}-\mathrm{e}}$ Different superscripts within the same column indicate significant differences among formulations $(p<0.05)$. 
reported when guar gum (galactomannan) was added to chitosan films, although further increase of concentration of galactomannan in the mixtures led to reduction of TS values (Rao et al., 2010). The addition of agar into cassava starch induced an improvement in EB and TS of cassava starch-based films (Phan The et al., 2009). The chemical interactions between $\kappa$-car and LBG could contribute for the obtained TS and EB results. It has been reported by our group that the addition of $\kappa$-car to LBG leads to stronger synergistic interactions (stronger gel) between the two polysaccharides, with a synergistic maximum in the viscoelastic properties for mixtures with a ratio of 60/40 $\kappa$-car/LBG (Pinheiro et al., 2011). However, in the present study it was the $40 / 60 \kappa$-car/LBG film the one which showed the best mechanical properties (TS and EB). These differences could be due to the addition of glycerol to the film-forming solution (in the preceding work no glycerol was added) and also to the different macromolecular behavior of film-forming mixtures when in aqueous solution and gel state. Other authors (Lafargue, Lourdin, \& Doublier, 2007) reported a strong influence of $\kappa$-car on the rheological behavior once in solution and in gel state of modified starch/ $\kappa$-car blends; however $\kappa$-car behavior on the film ("solid" state) was not noticeable.

Results showed that different polysaccharide ratios can be used to improve mechanical properties depending on the degree of interaction between the molecules of the components. The obtained values are in agreement with reported results for carrageenan and galactomannan-based films (Martins et al., 2010; Mikkonen et al., 2007; Sanchez-Garcia et al., 2010).

\subsection{Optical properties - color and opacity (OP) of the films}

In general, the films reported here present low opacity (OP) values (Table 3 ) and therefore good transparency, independently from the ratio $\kappa$-car/LBG used in film formation. Transparency is a valuable property in films once they are part of the packaging system and therefore interfere with consumers' choice. The results are in agreement with visual observation of the films, that present a clear and smooth appearance for all film formulations. Other authors (Li, Kennedy, Jiang, \& Xie, 2006) reported good miscibility between $40 \%$ konjac glucomannan-gelatin blend films, corresponding to the formulation providing the highest transparency. From OP results, there were no significant differences observed between the different ratios of the two polysaccharides used in the present work.

It terms of color, Table 3 shows that $L^{*}$ value obtained for $100 /$ $0 \kappa$-car/LBG films was not statistically different from the $0 / 100 \kappa$ car/LBG films. Additionally, $\kappa$-car/LBG films present a high $L^{*}$ value compared to other polysaccharide film blends e.g. presented by Rao et al. (2010) (48\% in the case of chitosan-guar gum films). The increase of LBG in the mixture $\kappa$-car/LBG did not affect the $L^{*}$ value (Table 3). The same behavior was reported by Rao et al. (2010) where the addition of $0 \%, 15 \%, 25 \%(v / v)$ guar gum to chitosan films did not change significantly the $L^{*}$ value. Overall, $a^{*}$ values of $\kappa$-car/LBG films suggest that these films tend to be reddish as

Table 3

Color parameters, $L^{*}, a^{*}$ and $b^{*}$, and opacity of the $\kappa$-car/LBG films.

\begin{tabular}{|c|c|c|c|c|}
\hline$\kappa-\operatorname{car} / \mathrm{LBG}(\% \mathrm{w} / \mathrm{w})$ & $L^{*}$ & $a^{*}$ & $b^{*}$ & Opacity (\%) \\
\hline $100 / 0$ & $96.95 \pm 0.35^{\mathrm{a}}$ & $5.29 \pm 0.07^{\mathrm{a}}$ & $-2.97 \pm 0.18^{\mathrm{a}}$ & $8.90 \pm 0.09^{\mathrm{ab}}$ \\
\hline $80 / 20$ & $96.71 \pm 0.30^{\mathrm{a}}$ & $5.15 \pm 0.04^{b}$ & $-2.46 \pm 0.26^{\mathrm{bc}}$ & $9.61 \pm 0.32^{\mathrm{a}}$ \\
\hline $60 / 40$ & $96.96 \pm 0.17^{\mathrm{a}}$ & $5.22 \pm 0.03^{\mathrm{ab}}$ & $-2.71 \pm 0.05^{c}$ & $8.97 \pm 0.62^{\mathrm{ab}}$ \\
\hline $40 / 60$ & $97.08 \pm 0.18^{\mathrm{a}}$ & $5.25 \pm 0.05^{a}$ & $-2.88 \pm 0.06^{\mathrm{a}}$ & $8.82 \pm 0.30^{\mathrm{b}}$ \\
\hline $20 / 80$ & $96.98 \pm 0.25^{\mathrm{a}}$ & $5.23 \pm 0.06^{\mathrm{ab}}$ & $-2.10 \pm 0.17^{b}$ & $9.55 \pm 0.09^{\mathrm{ab}}$ \\
\hline $0 / 100$ & $97.54 \pm 0.59^{\mathrm{a}}$ & $5.26 \pm 0.09^{\mathrm{ab}}$ & $-3.29 \pm 0.12^{\mathrm{e}}$ & $8.90 \pm 0.69^{\mathrm{ab}}$ \\
\hline
\end{tabular}

${ }^{\mathrm{a}-\mathrm{e}}$ Different superscripts within the same column indicate significant differences among formulations $(p<0.05)$. indicated by the positive $a^{*}$ values. When LBG was incorporated in $\kappa$-car (80/20 $\kappa$-car/LBG) mixture there was decrease of $a^{*}$ value; however, $a^{*}$ value increased when the percentage of LBG increased in the mixture (60/40 $\kappa$-car/LBG). This result could be due to films moisture content values. The increase in moisture content might change the reflection of light at the film surface, leading to film samples to be less red ( $a^{*}$ values decreased). Additionally, the results suggested that $0 / 100 \kappa$-car/LBG films present a higher intensification of the blueness comparing to the other mixture ratios (Table 3). Also, when $\kappa$-car or LBG are mix together, films presented a lower $b^{*}$ value. In any case, the values of $a^{*}$ and $b^{*}$ are of a magnitude that does not interfere with the color perception of most consumers.

\subsection{Principal component analysis (PCA)}

In order to select the best $\kappa$-car/LBG ratio film among the different film types with respect to their properties (good barrier, mechanical and optical properties) a PCA was carried out. The process of data analysis by PCA results in the formation of a score matrix and a loading matrix. The scores show the relationship between the samples in the new coordinate (PC defined) space, while the loadings illustrate the relationship between the original variables and the principal components (Vainionpää et al., 2004).

Fig. 6 presents the PCA of the first two principal components of $\kappa$-car/LBG films and shows how the different $\kappa$-car/LBG ratios affected film properties. The first two components (PC1 and PC2) together explained $80 \%$ of the variance, with PC1 and PC2 explaining $63 \%$ and $17 \%$ of the variance, respectively. Films that have little resemblance are situated far apart along each of the PCs, for example, 0/100 $\kappa$-car/LBG versus 40/60 $\kappa$-car/LBG or 0/100 $\kappa$ car/LBG versus 60/40 $\kappa$-car/LBG. PCA (Fig. 6) shows that WVP and EB are well correlated since they are plotted close to each other. This is in accordance to the previous results where different $\kappa$-car/ LBG ratios form a compact matrix reducing WVP and consequently, film flexibility. Whereas OP versus TS and moisture content (MC) loadings, plotted on opposite sides of the PC vector, are negatively correlated between each other. WVP and EB loadings which are placed orthogonally against TS and MC do not correlate; in fact, it is known that for increasing resistance of a film to deformation, lower

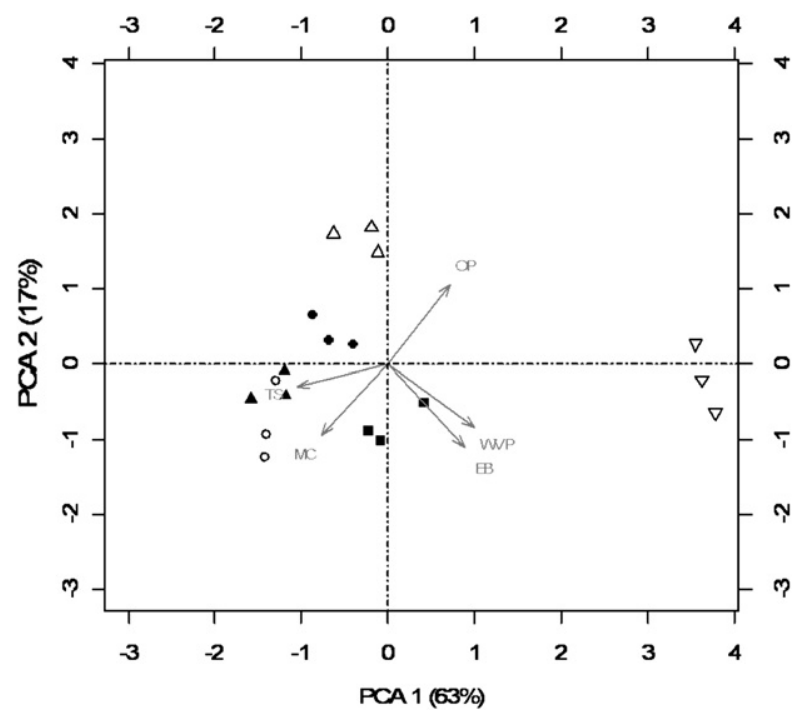

Fig. 6. Principal component analysis of the first two principal components of $\kappa$-car/

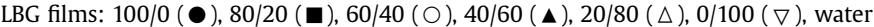
vapor permeability (WVP), moisture content (MC), opacity (OP), tensile strength (TS), and elongation-at-break (EB) of films. 
values of EB are registered. PC1 reflects the effects of film EB and WVP properties and showed mainly a positive correlation with EB and WVP and a negative correlation with TS and MC. PC2 is positively correlated with OP and negatively correlated with mechanical parameters (TS and EB), MC and WVP.

PCA showed that edible films composed of $40 / 60$ or $60 / 40$ of $\kappa-$ car/LBG are the best choice to be applied on food systems due to a good combination of water vapor barrier properties, mechanical and optical properties. However, when the properties of those two formulations (40/60 and 60/40 $\mathrm{\kappa}$-car/LBG) were compared (Table 2), 40/60 $\kappa$-car/LBG films present better mechanical and transport properties (higher values of TS and EB and lower WVP values) than 60/40 $\mathrm{\kappa}$-car/LBG films. Thus, the films composed of 40/ $60 \kappa$-car/LBG were elected as the films that should be applied as edible film for food packaging.

\section{Conclusion}

The results provided useful information on the structural properties of $\kappa$-car and LBG and the structural changes in the films network induced when mixing different ratios of the two polysaccharides. The synergistic effect between the two polymers, explained by miscibility between $\kappa$-car and LBG, was one the main reasons for the differences observed in the films properties. FTIR and TGA clearly show the good miscibility/compatibility of the two polymers for film production, since miscibility may be driven by the intermolecular interactions between polysaccharides. For all $\kappa$-car/ LBG films, the $40 / 60$ ratio films showed the best water vapor barrier and mechanical properties. The mixture of the two polysaccharides, besides allowing improvement of functionality (in terms of mechanical and water barrier properties) also allows tailoring films properties, at possibly lower costs due to the use of less expensive materials. These results contribute to the establishment of an approach to optimize films' composition based on the interactions between polysaccharides of different sources, aiming at improving polysaccharide-based films' properties when compared with other sources, and with non-edible and non-biodegradable films.

\section{Acknowledgments}

J. T. Martins, M. A. Cerqueira, A. I. Bourbon and A. C. Pinheiro gratefully acknowledge the Fundação para a Ciência e Tecnologia (FCT, Portugal) for their fellowships (SFRH/BD/32566/2006, SFRH/ BPD/72753/2010, SFRH/BD/73178/2010 and SFRH/BD/48120/2008, respectively), and B. W. S. Souza acknowledges the Coordenação de Aperfeiçoamento de Pessoal de Nível Superior (CAPES, Brazil). Prof. Cruz Pinto and Sandra Magina at CICECO-University of Aveiro are gratefully acknowledged for help with the DMA experiments.

\section{Appendix A. Supplementary data}

Supplementary material associated with this article can be found, in the online version, at doi:10.1016/j.foodhyd.2012.03.004.

\section{References}

Abugoch, L. E., Tapia, C., Villamán, M. C., Yazdani-Pedram, M., \& Díaz-Dosque, M. (2011). Characterization of quinoa protein-chitosan blend edible films. Food Hydrocolloids, 25(5), 879-886.

Arda, E., Kara, S., \& Pekcan, Ö (2009). Synergistic effect of the locust bean gum on the thermal phase transitions of $\kappa$-carrageenan gels. Food Hydrocolloids, 23, 451-459.

Avérous, L., Fringant, C., \& Moro, L. (2001). Plasticized starch-cellulose interactions in polysaccharide composites. Polymer, 42(15), 6565-6572.

Biliaderis, C. G., Lazaridou, A., \& Arvanitoyannis, I. (1999). Glass transition and physical properties of polyol-plasticised pullulan-starch blends at low moisture. Carbohydrate Polymers, 40, 29-47.
Bourtoom, T. (2008). Edible films and coatings: characteristics and properties. International Food Research Journal, 15(3), 1-12.

Casariego, A., Souza, B. W. S., Cerqueira, M. A., Cruz, L., Díaz, R., \& Vicente, A. A. (2009). Chitosan/clay films' properties as affected by biopolymer and clay micro/nanoparticles' concentrations. Food Hydrocolloids, 23, 1895-1902.

Cerqueira, M. A., Souza, B. W. S., Simões, J., Teixeira, J. A., Domingues, M. R. R. M., Coimbra, M. A., et al. (2011). Structural and thermal characterization of galactomannans from non-conventional sources. Carbohydrate Polymers, 83(1) 179-185.

Cerqueira, M. A., Souza, B. W. S., Teixeira, J. A., \& Vicente, A. A. (2012). Effect of glycerol and corn oil on physicochemical properties of polysaccharide films a comparative study. Food Hydrocolloids, 27, 175-184.

Cha, D. S., Choi, J. H., Chinnan, M. S., \& Park, H. J. (2002). Antimicrobial films based on Na-alginate and $\kappa$-carrageenan. LWT - Food Science and Technology, 35, 715-719.

Cherian, G., Gennadios, A., Weller, C. L., \& Chinachoti, P. (1995). Thermomechanical behavior of wheat gluten films: effect of sucrose, glycerin, and sorbitol. Cereal Chemistry, 72(1), 1-6.

Cunha, P. L. R., Castro, R. R., Rocha, F. A. C., de Paula, R. C. M., \& Feitosa, J. P. A. (2005) Low viscosity hydrogel of guar gum: preparation and physicochemical characterization. International Journal of Biological Macromolecules, 37(1-2), 99-104.

Dakia, P. A., Blecker, C., Robert, C., Wathelet, B., \& Paquot, M. (2008). Composition and physicochemical properties of locust bean gum extracted from whole seeds by acid or water dehulling pre-treatment. Food Hydrocolloids, 22, 807-818.

Duncan, J. (2008). Principles and applications of mechanical thermal analysis. In P. Gabbott (Ed.), Principles and applications of thermal analysis (pp. 119-163). Oxford: Wiley-Blackwell.

Figueiró, S. D., Góes, J. C., Moreira, R. A., \& Sombra, A. S. B. (2004). On the physicochemical and dielectric properties of glutaraldehyde crosslinked galactomannan-collagen films. Carbohydrate Polymers, 56(3), 313-320.

Garcia, M. A., Pinotti, A., \& Zaritzky, N. E. (2006). Physicochemical, water vapor barrier and mechanical properties of corn starch and chitosan composite films. Starch, 58, 453-463.

García-García, E., \& Totosaus, A. (2008). Low-fat sodium-reduced sausages: effect of the interaction between locust bean gum, potato starch and $\kappa$-carrageenan by a mixture design approach. Meat Science, 78, 406-413.

Johnson, J. L., Busk, G. B., \& Labuza, T. P. (1980). Examination of the crystallinity of food gels by X-ray diffraction. Journal of Food Science, 45, 77-83.

Jones, D. S. (1999). Dynamic mechanical analysis of polymeric systems of pharmaceutical and biomedical significance. International Journal of Pharmaceutics, $179,167-178$

Karbowiak, T., Debeaufort, F., Champion, D., \& Voilley, A. (2006). Wetting properties at the surface of iota-carrageenan-based edible films. Journal of Colloid and Interface Science, 294, 400-410.

Karbowiak, T., Hervet, H., Léger, L., Champion, D., Debeaufort, F., \& Voilley, A. (2006) Effect of plasticizers (water and glycerol) on the diffusion of a small molecule in iota-carrageenan biopolymer films for edible coating application. Biomacromolecules, 7(6), 2011-2019.

Khwaldia, K., Arab-Tehrany, E., \& Desobry, S. (2010). Biopolymer coatings on paper packaging materials. Comprehensive Reviews in Food Science and Food Safety, 9, 82-91.

Lafargue, D., Lourdin, D., \& Doublier, J.-L. (2007). Film-forming properties of a modified starch/ $/$-carrageenan mixture in relation to its rheological behavior Carbohydrate Polymers, 70, 101-111.

Li, B., Kennedy, J. F., Jiang, Q. G., \& Xie, B. J. (2006). Quick dissolvable, edible and heatsealable blend films based on konjac glucomannan - gelatin. Food Research International, 39, 544-549.

Li, B., Li, J., Xia, J., Kennedy, J. F., Yie, X., \& Liu, T. G. (2011). Effect of gamma irradiation on the condensed state structure and mechanical properties of konjac glucomannan/chitosan blend films. Carbohydrate Polymers, 83(1), 44-51.

Liu, X., Dever, M., Fair, N., \& Benson, R. S. (1997). Thermal and mechanical properties of poly(lactic acid) and poly(ethylene/butylene succinate) blends. Journal of Environmental Polymer Degradation, 5(4), 225-235.

Mano, J. F. (2005). Viscoelastic properties of bone: mechanical spectroscopy studies on a chicken model. Materials Science and Engineering C, 25, 145-152.

Martins, J. T., Cerqueira, M. A., Souza, B. W. S., Avides, M. C., \& Vicente, A. A. (2010) Shelf life extension of ricotta cheese using coatings of galactomannans from nonconventional sources incorporating nisin against Listeria monocytogenes. Journal of Agricultural and Food Chemistry, 58(3), 1884-1891.

Mathew, A. P., \& Dufresne, A. (2002). Plasticized waxy maize starch: effect of polyols and relative humidity on material properties. Biomacromolecules, 3, 1101-1108.

Mikkonen, K. S., Rita, H., Helén, H., Talja, R. A., Hyvönen, L., \& Tenkanen, M. (2007) Effect of polysaccharide structure on mechanical and thermal properties of galactomannan-based films. Biomacromolecules, 8, 3198-3205.

Ning, W., Jiugao, Y., Xiaofei, M., \& Ying, W. (2007). The influence of citric acid on the properties of thermoplastic starch/linear low-density polyethylene blends. Carbohydrate Polymers, 67(3), 446-453.

Parker, A., Lelimousin, D., Miniou, C., \& Boulenguer, P. (1995). Binding of galactomannans to kappa-carrageenan after cold mixing. Carbohydrate Research, 272, 91-96.

Pelissari, F. M., Grossmann, M. V. E., Yamashita, F., \& Pineda, E. A. G. (2009). Antimicrobial, mechanical, and barrier properties of cassava starch-chitosan films incorporated with oregano essential oil. Journal of Agricultural and Food Chemistry, 57, 7499-7504. 
Pereira, L., Amado, A. M., Critchley, A. T., van de Velde, F., \& Ribeiro-Claro, P. J. A. (2009). Identification of selected seaweed polysaccharides (phycocolloids) by vibrational spectroscopy (FTIR-ATR and FT-Raman). Food Hydrocolloids, 23(7), 1903-1909.

Pereira, R. N., Souza, B. W. S., Cerqueira, M. A., Teixeira, J. A., \& Vicente, A. A. (2010). Effects of electric fields on protein unfolding and aggregation: influence on edible films formation. Biomacromolecules, 11(11), 2912-2918.

Phan The, D., Debeaufort, F., Voilley, A., \& Luu, D. (2009). Biopolymer interactions affect the functional properties of edible films based on agar, cassava starch and arabinoxylan blends. Journal of Food Engineering, 90(4), 548-558.

Pinheiro, A. C., Bourbon, A. I., Rocha, C., Ribeiro, C., Maia, J. M., Gonçalves, M. P., et al. (2011). Rheological characterization of [kappa]-carrageenan/galactomannan and xanthan/galactomannan gels: comparison of galactomannans from nontraditional sources with conventional galactomannans. Carbohydrate Polymers, 83(2), 392-399.

Pollard, M. A., Kelly, R., Wahl, C., Fischer, P., Windhab, E., Eder, B., et al. (2007) Investigation of equilibrium solubility of a carob galactomannan. Food Hydrocolloids, 21, 683-692.

Prasad, K., Kaneko, Y., \& Kadokawa, J. (2009). Novel gelling systems of $\kappa-$, - - and $\lambda$ carrageenans and their composite gels with cellulose using ionic liquid. Macromolecular Bioscience, 9, 376-382.

Rao, M. S., Kanatt, S. R., Chawla, S. P., \& Sharmam, A. (2010). Chitosan and guar gum composite films: preparation, physical, mechanical and antimicrobial properties. Carbohydrate Polymers, 82(4), 1243-1247.

Rees, D. A. (1981). Polysaccharide shapes and their interactions - some recent advances. Pure and Applied Chemistry, 53(1), 1-14.

Sanchez-Garcia, M. D., Hilliou, L., \& Lagaron, J. M. (2010). Nanobiocomposites of carrageenan, zein, and mica of interest in food packaging and coating applications. Journal of Agricultural and Food Chemistry, 58(11), 6884-6894.

Sen, M., \& Erboz, E. N. (2010). Determination of critical gelation conditions of [kappa]-carrageenan by viscosimetric and FT-IR analyses. Food Research International, 43(5), 1361-1364.

Soares, R. M. D., Lima, A. M. F., Oliveira, R. V. B., Pires, A. T. N., \& Soldi, V. (2005). Thermal degradation of biodegradable edible films based on xanthan and starches from different sources. Polymer Degradation and Stability, 90(3), $449-454$.

Souza, B., Cerqueira, M., Teixeira, J., \& Vicente, A. (2010). The use of electric fields for edible coatings and films development and production: a review. Food Engineering Reviews, 2(4), 244-255.

Spagnuolo, P. A., Dalgleish, D. G., Goff, H. D., \& Morris, E. R. (2005). Kappa-carrageenan interactions in systems containing casein micelles and polysaccharide stabilizers. Food Hydrocolloids, 19, 371-377.

Tako, M., Qi, Z.-Q., Yoza, E., \& Toyama, S. (1998). Synergistic interaction between $\kappa$ carrageenan isolated from Hypnea charoides Lamouroux and galactomannan on its gelation. Food Research International, 31(8), 543-548.

Thành, T. T. T., Yuguchi, Y., Mimura, M., Yasunaga, H., Takano, R., Urakawa, H., et al. (2002). Molecular characteristics and gelling properties of the carrageenan family, 1. Preparation of novel carrageenans and their dilute solution properties. Macromolecular Chemistry and Physics, 203, 15-23.
Turquois, T., Acquistapace, S., Vera, F. A., \& Welti, D. H. (1996). Composition of carrageenan blends inferred from 13C-NMR and infrared spectroscopic analysis. Carbohydrate Polymers, 31(4), 269-278.

Vainionpää, J., Smolander, M., Alakomi, H.-L., Ritvanen, T., Rajamäki, T., Rokka, M., et al. (2004). Comparison of different analytical methods in the monitoring of the quality of modified atmosphere packaged broiler chicken cuts using principal component analysis. Journal of Food Engineering, 65, 273-280.

van de Velde, \& de Ruiter, G. A. (2005). Carrageenan. In A. Steinbüchel, \& S. K. Rhee (Eds.), Polysaccharides and polyamides in the food industry, Vol. 1 (pp. 85-114). Weinheim: Wiley-VCH.

Vendruscolo, C. W., Ferrero, C., Pineda, E. A. G., Silveira, J. L. M., Freitas, R. A., Jiménez-Castellanos, M. R., et al. (2009). Physicochemical and mechanical characterization of galactomannan from Mimosa scabrella: effect of drying method. Carbohydrate Polymers, 76, 86-93.

Villetti, M., Crespo, J., Soldi, M., Pires, A., Borsali, R., \& Soldi, V. (2002). Thermal degradation of natural polymers. Journal of Thermal Analysis and Calorimetry, 67(2), 295-303.

Vittadini, E., \& Vodovotz, Y. (2003). Changes in the physicochemical properties of wheat- and soy-containing breads during storage as studied by thermal analyses. Journal of Food Science, 68(6), 2022-2027.

Wanchoo, R. K., \& Sharma, P. K. (2003). Viscometric study on the compatibility of some water-soluble polymer-polymer mixtures. European Polymer Journal, 39(7), 1481-1490.

Watanabe, T., Ohtsuka, A., Murase, N., Barth, P., \& Gersonde, K. (1996). NMR studies on water and polymer diffusion in dextran gels. Influence of potassium ions on microstructure formation and gelation mechanism. Magnetic Resonance in Medicine, 35(5), 697-705.

Williams, P. A. (2009). Molecular interactions of plant and algal polysaccharides. Structural Chemistry, 20, 299-308.

Williams, P. A., \& Langdon. (1996). The influence of locust bean gum and dextran on the gelation of $\kappa$-carrageenan. Biopolymers, 38, 655-664.

Xu, J. B., Bartley, J. P., \& Johnson, R. A. (2003). Preparation and characterization of alginate-carrageenan hydrogel films crosslinked using a water-soluble carbodiimide (WSC). Journal of Membrane Science, 218, 131-146.

Xu, X., Li, B., Kennedy, J. F., Xie, B. J., \& Huang, M. (2007). Characterization of konjac glucomannan-gellan gum blend films and their suitability for release of nisin incorporated therein. Carbohydrate Polymers, 70, 192-197.

Yakimets, I., Paes, S. S., Wellner, N., Smith, A. C., Wilson, R. H., \& Mitchell, J. R. (2007). Effect of water content on the structural reorganization and elastic properties of biopolymer films: a comparative study. Biomacromolecules, 8, 1710-1722.

Zhong, Q.-P., \& Xia, W.-S. (2008). Physicochemical properties of edible and preservative films from chitosan/cassava starch/gelatin blend plasticized with glycerol. Food Technology and Biotechnology, 46(3), 262-269.

Ziani, K., Oses, J., Coma, V., \& Maté, J. I. (2008). Effect of the presence of glycerol and Tween 20 on the chemical and physical properties of films based on chitosan with different degree of deacetylation. LWT - Food Science and Technology, 41(10), 2159-2165.

Zohuriaan, M. J., \& Shokrolahi, F. (2004). Thermal studies on natural and modified gums. Polymer Testing, 23(5), 575-579. 